\title{
A REVISION OF GENTIANELLA,COMASTOMA AND GENTIANOPSIS (GENTIANACEAE) IN NEPAL
}

\author{
E. AITKEN
}

The genus Gentianella in Nepal is revised and five species are recognized: G. angustiflora Harry Sm., G. azurea (Bunge) Holub, G. glanduligera Airy Shaw, G. lowndesii Harry Sm. and G. moorcroftiana (Wall. ex G.Don) Airy Shaw. Gentianella maddenii (C.B.Clarke) Airy Shaw, usually treated as a distinct species, is placed in synonymy of $G$. moorcroftiana. The genus Comastoma in Nepal is revised, four species are recognized and one new combination is made: $C$. falcatum (Turcz. ex Kar. \& Kir.) Toyok., $C$. pedunculatum (D.Don) Holub, C. stellariifolium (Franch.) Holub and C. urnigerum (E.Aitken \& D.G.Long) E.Aitken comb. nov. The genus Gentianopsis in Nepal is revised and three species are recognized: G. contorta (Royle) Ma, G. paludosa (Hook.) Ma and G. vvedenskyi (Grossh.) Pissjauk.

Keywords. Comastoma, Gentianella, Gentianopsis, Nepal, taxonomy.

\section{INTRODUCTION}

In the revision of Gentianaceae for the Flora of Bhutan Vol. 2 Part 2 (Aitken, 1999), the genus Gentianella was treated in the broad sense, to include Comastoma, Aloitis, Aliopsis and Gentianopsis, as it seemed an appropriate arrangement for such a localized study and also followed the herbarium arrangement at E, K and BM (see Aitken \& Long, 1994). This led to occasional problems as to which generic names were appropriate in studies covering wider geographical areas as there is no generally accepted worldwide treatment of generic limits. Von Hagen \& Kadereit (2001) have found good molecular evidence to separate Comastoma from Gentianella s.str. However, their findings also indicate that the relationships between Gentianella s.str., Comastoma, Gentianopsis, Lomatogonium and Swertia p.p. are very complex and further sampling is required to obtain more clearly defined groupings.

The Checklist of the Flowering Plants of Nepal (Press et al., 2000) and the Flora of China (Ho \& Pringle, 1995) have treated Comastoma and Gentianopsis as separate genera from Gentianella, and the same treatment is followed here. This also makes it necessary to transfer Gentianella urnigera to the genus Comastoma, making it Comastoma urnigerum (E.Aitken \& D.G.Long) E.Aitken.

Notes. Geographical distributions are cited in accordance with the forthcoming Flora of Nepal: e.g. W corresponds to Mid Western and Far Western Development

Royal Botanic Garden Edinburgh, 20A Inverleith Row, Edinburgh EH3 5LR, UK. E-mail: e.aitken@rbge.ac.uk 
Regions, $\mathbf{C}$ to Central and Western Development Regions and $\mathbf{E}$ to Eastern Development Region.

All specimens have been seen unless otherwise indicated.

\section{Key to the genera}

1a. Corolla with fringe of thick, white, non-vascularized fimbriae in throat

2. Comastoma

1b. Corolla without fringe of fimbriae in throat 2

2a. Calyx tubular, 4-angled, lobes in unequal pairs; intracalycular membrane present

3. Gentianopsis

2b. Calyx divided almost to base, lobes sometimes unequal but not in pairs; intracalycular membrane absent

1. Gentianella

1. Gentianella Moench, Meth. P1. 482 (1794)

Annual, biennial or perennial herbs. Leaves opposite. Flowers 4- or 5-merous, terminal, solitary or in cymes. Calyx divided almost to base, without intracalycular membrane. Corolla tubular, subcampanulate or funnel-shaped, without appendages or plicae. Nectaries 8 or 10 at base of corolla tube, double the number of corolla lobes. Stamens attached at middle or base of corolla tube. Ovary sessile or with distinct stipe. Stigmas 2, prominent, sessile or subsessile. Capsules 2-valved. Seeds smooth to warty.

About 125 species: $\mathrm{N}$ and $\mathrm{S}$ temperate zones worldwide; five species in Nepal. - Type species: Gentianella tetranda Moench, based on Gentiana campestris L. = Gentianella campestris (L.) Börner.

\section{Key to species of Gentianella}

1a. Flowers tubular, in elongated, narrow cymes; calyx usually less than half corolla length

1. G. angustiflora

1b. Flowers funnel-shaped, hypocrateriform or subcampanulate; calyx more than half corolla length

2a. Flowers hypocrateriform or subcampanulate; calyx with distinct blackish margin; ovary sessile

2. G. azurea

2b. Flowers funnel-shaped; calyx without blackish margin; ovary not sessile 3

3a. Lower leaves elliptic; flowers angled; corolla lobes very short, about $1 / 4$ length of tube

4. G. lowndesii

3b. Lower leaves lanceolate or linear; flowers not angled; corolla lobes more than $1 / 4$ length of tube 
4a. Leaves linear, 5-10 × 1-2 mm; calyx tube 1-2 mm; corolla 9-12 mm long

3. G. glanduligera

4b. Leaves linear to lanceolate, 6-20(-30) $\times 1-4 \mathrm{~mm}$; calyx tube usually more than $2 \mathrm{~mm}$; corolla (9-)11-27 mm long 5. G. moorcroftiana

1. Gentianella angustiflora Harry Sm., [ex Nilsson in Grana Palyn. 7: 106 (1967), nom. nud.] J. Jap. Bot. 56: 275 (1981). - Type: C Nepal: Manang district, Sabze Khola, 13,000 ft, 5 ix 1950, D. G. Lowndes 1490 (holo BM). Fig. 1.

Annual to $25 \mathrm{~cm}$ tall. Stems erect or slightly decumbent, often reddish, angular or ridged, scabrous. Basal leaves withered at flowering time; stem leaves sometimes reddish, lanceolate to elliptic, 4-12 × 1-3 mm, acute. Inflorescence of elongated, narrow cymes. Pedicel 3-12 mm. Flowers 4- or 5-merous, often angled up to $90^{\circ}$ on pedicel. Calyx scabrous, green or reddish; tube c. $1 \mathrm{~mm}$; lobes unequal, lanceolate, $2-4 \times 0.5-1 \mathrm{~mm}$, slightly spreading, acute, sometimes narrowed at base. Corolla dark blue or purple, narrowly tubular; tube $4-8 \mathrm{~mm}$; lobes oblong or ovate, $2-3 \times$ $0.5-2 \mathrm{~mm}$, subacute or rounded. Filaments $1-1.5 \mathrm{~mm}$; anthers yellow, ellipsoid,


F IG. 1. Gentianella angustiflora Harry Sm. A, calyx; B, corolla; C, ovary; D, habit. A, B \& C based on Polunin, Sykes \& Williams 5365; D based on Stainton, Sykes \& Williams 5310. Scale bars: $1 \mathrm{~mm}(\mathrm{~A}-\mathrm{C}), 1 \mathrm{~cm}(\mathrm{D})$. 
c. $0.5 \mathrm{~mm}$. Ovary sessile, narrowly ellipsoid, 3-7 $\times 1-2 \mathrm{~mm}$. Style indistinct; stigma lobes suborbicular. Capsule 6-11 $\times 1-2 \mathrm{~mm}$, protruding slightly from corolla. Seeds smooth.

Distribution. W, C and E Nepal [W Himalaya (Kashmir); Tibetan Plateau (China: Xizang)].

Ecology. Open grass slopes or in sand among rocks on river bed; $1220-4570 \mathrm{~m}$. Flowering and fruiting August-September.

Specimens examined. W: Dolpa district, 4 miles west of Tingkyu, c.4200 m, 5 xiii 1973, GreyWilson \& Phillips 509 (K); Lulo Khola, 15,000 ft, 19 ix 1952, Polunin, Sykes \& Williams 3516 (BM, E); Jangla Banyang, 3800 m, 18 viii 1973, Einarsson, Skärby \& Wetterhall 3568 (BM). Mugu district, Khaptang, Mugu Khola, 15,000 ft, 21 viii 1952, Stainton, Sykes \& Williams 5363 (BM, E). Rukum district, East of Chalike Pahar, 4000 ft, 21 ix 1954, Stainton, Sykes \& Williams 4523 (BM, E). C: Mustang district, Bhena, $28^{\circ} 59^{\prime} \mathrm{N}, 83^{\circ} 48^{\prime} \mathrm{E}, 3780 \mathrm{~m}, 26$ viii 2001, $G$. \& S. Miehe 01-072-24 (E); Pass between Narsing and Yak Khola, $28^{\circ} 54^{\prime} \mathrm{N}, 83^{\circ} 58^{\prime} \mathrm{E}, 4770 \mathrm{~m}, 8$ viii 2001, G. \& S. Miehe 01-017-22 (E); Sandah, north of Tukucha, 13,500 ft, 18 viii 1954, Stainton, Sykes \& Williams 7310 (BM, E). Manang district, north aspect of Annapurna, 3900 m, 28 ix 2004, Chaudhary, Bhatta \& Subedi 490 (TUCH, photocopy at E). E: Sankhuwasabha district, Nehe Kharka, S side of Barun Khola, 3730 m, 30 ix 1991, EMAK 430 (E).

Note. Garg (1987) made this a synonym of G. azurea (Bunge) Holub. Although the flowers of $G$. azurea are also angled on the pedicels it has several distinct differences, described below.

2. Gentianella azurea (Bunge) Holub, Folia Geobot. Phytotax. 2: 116 (1967). - Gentiana azurea Bunge, Nouv. Mém. Soc. Nat. Mosc. 7: 230, t.10, fig.3 (1829). - Aloitis azurea (Bunge) Omer, Fl. Pakistan 197: 109 (1995). - Type: Russia, Siberia, Baikal, 1821, Bunge s.n. (holo LE, digital image; iso G-DC, microfiche).

Annual to $7 \mathrm{~cm}$ tall. Stems ascending, branched diffusely from base and above, ridged, scabrous. Basal leaves often withered at flowering time; stem leaves ovate to lanceolate, 3-7 × 2-3 mm, acute, sometimes with blackish margin. Inflorescence of loose, few-flowered cymes. Pedicel 10-40 mm. Flowers 5-merous. Calyx tube 1-2 mm; lobes elliptic to lanceolate, 3-4 mm, with distinct blackish margin. Corolla deep blue, subcampanulate or hypocrateriform; tube 4-5 mm; lobes ovate, 3-4 × 1-2 mm, acute. Filaments 2-3 mm; anthers blue, c. $1 \mathrm{~mm}$. Ovary sessile, narrowly ellipsoid, 6-7 $\times 1 \mathrm{~mm}$. Style indistinct; stigma lobes suborbicular. Capsule not seen.

Distribution. W Nepal [Tibetan Plateau (Qinghai, Xinjiang, Xizang); E Himalaya (Bhutan); W Himalaya (Pakistan); E Asia (China: Gansu, NW Sichuan, NW Yunnan); C and N Asia (Kazakhstan, Kyrgyzstan, Mongolia, Siberia)]. Not previously recorded from Nepal.

Ecology. Open hillsides; c.5000 m. Flowering August. 
Specimens examined. W: Dolpa district, West side of Khung Khola, c.5000 m, 19 xiii 1973, Grey-Wilson \& Phillips 678 (2 sheets, K).

Note. Gentiana marginata Turcz. ex Bess. has been included as a synonym of $G$. azurea by various authors but no type specimen is available to allow the validity of this to be assessed.

3. Gentianella glanduligera Airy Shaw in Hooker's Icon. Pl. 35: t.3431 (1943). - Gentianella maddenii var. glanduligera (Airy Shaw) S.Agrawal, Indian Forester 109: 576 (1983). - Type: Cult. ex Nepal. Grown in Mr. C. T. Musgrave's garden at Hascombe, near Godalming, Surrey from seeds sent by Mr. Hay, Oct. 1939 "Seed no. 69" (holo K).

Ascending or erect annual to $11 \mathrm{~cm}$ tall. Stems branched from base and above, purplish red. Leaves green above, often slightly purplish beneath, linear, 5-10 $\times$ 1-2 mm, acute, bases shortly connate and minutely scaberulous-papillose. Inflorescence of loose cymes. Pedicel 5-20 mm. Flowers 5-merous. Calyx tube 1-2 mm, purplish; lobes green within, purple outside, linear to subspathulate, 4-7 $\times$ 1-2 mm, acute, slightly fleshy, minutely papillose, apex spreading. Corolla funnelshaped; tube green at base, 5-7 mm, whitish inside; lobes purple to greenish blue outside, bright purplish blue inside, oblong, 4-5 $\times 1-2.5 \mathrm{~mm}$, obtuse or subacute, contorted in bud. Filaments $5-6 \mathrm{~mm}$; anthers deep purple, $0.5 \mathrm{~mm}$. Ovary narrowly ellipsoid or oblong, 5-7.5 × 1-2 mm; stipe 0.5-0.75 mm. Style indistinct or c. $1 \mathrm{~mm}$. Stigma ligulate, bluish, $1.5 \mathrm{~mm}$, densely papillose, shortly decurrent on ovary, recurved. Capsule not seen.

Distribution. C Nepal [W Himalaya (India: Kumaon?)].

Ecology. Open grassy hillsides; 2800-4575 m. Flowering September-October.

Specimens examined. C: Manang district, Khangsar, 15,000 ft, 9 xi 1950, D. G. Lowndes 1503 (BM); Marsyandi valley: on alluvions above Chame, 2800 m, 24 ix 1969, T. Wraber 386 (BM). Mustang district, E of Thorong-La, 14,200 ft, 9 xi 1977, G. Miehe 642 (BM); Chalungpa, above Jelua Forest, $28^{\circ} 53^{\prime} \mathrm{N}, 83^{\circ} 44^{\prime} \mathrm{E}, 4350 \mathrm{~m}, 6$ ix 2001, G. \& S. Miehe 01-111-07a (E); Chalungpa, above Jelua Forest, $28^{\circ} 53^{\prime} \mathrm{N}, 8^{\circ} 44^{\prime} \mathrm{E}, 4350 \mathrm{~m}, 6$ ix 2001, G. \& S. Miehe 01-111-06 (E).

Note. As Gentianella glanduligera was originally described from cultivated material, there seems to have been some doubt regarding its status as a species. Airy Shaw (1943) recognized a close relationship with Gentianella maddenii. Agrawal (1983) considered it to be a cultivated form of G. maddenii and reduced it to Gentianella maddenii var. glanduligera (Airy Shaw) S.Agrawal. She based this on one specimen, T.A. Rao 4589 (BSD) from Kumaon, the identity of which has not been verified here. Depauperate forms of Gentianella maddenii or $G$. moorcroftiana differ from $G$. glanduligera in having proportionally much larger, elongated flowers in relation to the plant size. 
4. Gentianella lowndesii Harry Sm., J. Jap. Bot. 56: 276 (1981). - Type: C Nepal: Manang district, Bimtakothi, D. G. Lowndes 1541 (holo BM).

Erect biennial to $20 \mathrm{~cm}$ tall. Stems simple or with few branches, sometimes reddish. Leaves lanceolate, 3-20 × 1-4 mm, acute, sometimes dark reddish purple; upper stem leaves often whorled and larger than lower leaves. Inflorescence one-flowered or of loose, few-flowered cymes. Pedicel 6-40 mm. Flowers 5-merous, angled on pedicel up to $90^{\circ}$ when mature. Calyx tube $3-5 \mathrm{~mm}$; lobes lanceolate to spathulate, slightly unequal, 4-10 × 1-3 mm, acute, narrowing at base. Corolla funnel-shaped, purplish blue, red-violet or lilac, sometimes with darker stripes; tube (8-)10-18 mm; lobes oblong or ovate, $2-4 \times 2-4 \mathrm{~mm}$, rounded or obtuse. Filaments $(4-) 8-10 \mathrm{~mm}$; anthers 1-1.5 mm. Ovary cylindrical, (5-)9-10 × (1-)2-3 mm; stipe (2-)3-4 mm. Style absent. Stigmas flattened, c.1 mm, recurved. Capsule not seen.

Distribution. Endemic to W and C Nepal.

Ecology. Glacial moraine, rock ledges or silt; 3800-4570 m. Flowering AugustSeptember.

Specimens examined. W: Dolpa district, Lulo Khola, 15,000 ft, 18 ix 1952, Polunin, Sykes \& Williams 3479 (BM, E); Rohagaon, Suli Gad, 11,000 ft, 14 ix 1952, Polunin, Sykes \& Williams 3382 (mixed sheet with G. moorcroftiana) (BM). Jumla district, Munigaon, 3800 m, 27 viii 1973, Einarsson, Skärby \& Wetterhall 3725 (BM). C: Manang district, Bimtakothi, 13,500 ft, 27 viii 1950, Lowndes 1470 (BM).

5. Gentianella moorcroftiana (Wall. ex G.Don) Airy Shaw in Hooker's Icon. P1. 35: sub t.3431 (1943). - Gentiana moorcroftiana Wall. [Cat.154, n.4390 (1831), nom. nud.] ex G.Don, Gen. Syst. 4: 182 (1838). - Aloitis moorcroftiana (Wall. ex G.Don) Omer, Qaiser \& Ali, Pakistan J. Bot. 20: 57 (1988). - Type: From Srinuggar to Luddak, Moorcroft in Wallich 4390 (lecto K-W, first step designation by Omer in Fl. Pakistan 197: 115 (1995), second step, central plant of 5 on sheet, designated here; iso BM, E, K n.v., K-W, digital image).

Gentiana moorcroftiana var. maddenii C.B.Clarke in Hook.f., Fl. Brit. Ind. 4: 108 (1883). - Gentianella maddenii (C.B.Clarke) Airy Shaw in Hooker's Icon. P1. 35: sub t.3431 (1943). - Aloitis maddenii (C.B.Clarke) Omer, Qaiser \& Ali, Pakistan J. Bot. 20: 155 (1988). - Type: India: Kumaon, Pinsara Pass, 3270 m, x 1875, Davidson s.n. (lecto K, designated here; iso DD n.v.).

Erect annual or biennial to $22 \mathrm{~cm}$ tall. Stems, leaves and calyx glabrous or sometimes reddish and finely glandular-papillose. Stems single or diffusely branched from base or above. Lower leaves withered at flowering time; stem leaves linear to lanceolate, 6-20(-30) $\times 1-4 \mathrm{~mm}$, acute. Inflorescence of many-flowered cymes. Pedicel (2-)520(-30) mm. Flowers 5-merous. Calyx tube 2-5 mm; lobes unequal, linear to lanceolate, (2-)4-20 × 0.5-2 mm, acute or rounded, sometimes reflexed at apex or narrowed at base. Corolla mauve, blue or pink, often white within; funnel-shaped or 
tubular, tube $6-15 \mathrm{~mm}$, lobes oblong to obovoid, $(3-) 5-12 \times 2-5 \mathrm{~mm}$, rounded or acute. Filaments $2-6 \mathrm{~mm}$; anthers 1-1.5 mm. Ovary narrowly ellipsoid, $8-15 \times 1-3 \mathrm{~mm}$; stipe 1-3 mm. Style c.1 mm. Stigma c. $1 \mathrm{~mm}$. Capsule not seen.

Distribution. W and C Nepal [Tibetan Plateau (Xizang); W Himalaya (Pakistan, India, Kashmir); SW Asia (Afghanistan)].

Ecology. Open grass slopes or scree; 2890-5180 m. Flowering August-October.

Specimens examined. W: Bajhang district, 4 miles NE of Saipal, 17,000 ft, 26 viii 1954, J.E.M. Arnold 269 (BM). Dolpa district, Rohagaon, Suli Gad, 11,000 ft, 14 ix 1952, Polunin, Sykes \& Williams 3382 (mixed sheet with G. lowndesii) (BM); Rohagaon, Suli Gad, 11,500 ft, 14 ix 1952, Polunin, Sykes \& Williams 3393 (BM, E); North-east of Tarap, c.4500 m, 27 viii 1973, Grey-Wilson \& Phillips 750 (K). Jumla district, Munigaon, SE of Jumla, 9500 ft, 28 ix 1952, Polunin, Sykes \& Williams 5445 (BM, E); Between Padmara and Jumla, 10,500 ft, 6 x 1952, Polunin, Sykes \& Williams 5508 (BM, E); Chakura Lekh, 14,000ft, 29 ix 1952, Polunin, Sykes \& Williams 5691 (BM). Rukum district, East of Chalike Pahar, 14,000 ft, 23 ix 1954, Stainton, Sykes \& Williams 4566 (BM, E). C: Mustang district, Muktinath, 13,500 ft, 1 x 1954, Stainton, Sykes \& Williams 8056 (BM); Muktinath, 13,000 ft, 1 x 1954, Stainton, Sykes \& Williams 8133 (BM, E); Larjung, south of Tukucha, Kali Gandaki valley, $9500 \mathrm{ft}, 16$ x 1954, Stainton, Sykes \& Williams 8172 (BM, E); Tukucha, Kali Gandaki valley, 10,000 ft, 15 x 1954, Stainton, Sykes \& Williams 8155 (BM, E); Chalungpa, above Jelua Forest, $28^{\circ} 53^{\prime} \mathrm{N}, 83^{\circ} 44^{\prime} \mathrm{E}, 4350 \mathrm{~m}, 6$ ix 2001, G. \& S. Miehe 01-111-07 (E). District uncertain: Near Saure Khola, 14,500 ft, 3 x 1954, Stainton, Sykes \& Williams 4679 (BM, E).

Notes. The above synonymy is in accordance with Ho \& Pringle (1995). This is an extremely variable species which may occur in very large or dwarf forms which intergrade. Specimens with narrower leaves and more tubular corollas have been treated separately by Clarke (1883) as Gentiana moorcroftiana var. maddenii and by Airy Shaw (1943) as Gentianella maddenii.

\section{Comastoma (Wettst.) Toyok., Bot. Mag. (Tokyo) 74: 198 (1961)}

Annual or perennial herbs. Basal leaves in rosette, stem leaves opposite. Flowers 4or 5-merous, solitary on long pedicels or in loose cymes. Calyx divided almost to base, without intracalycular membrane. Corolla tubular, funnel-shaped or urnshaped; throat fringed with thick, non-vascularized, white fimbriae; plicae absent. Nectaries at base of corolla tube 8 or 10 , double the number of corolla lobes. Stamens attached at middle or towards base of corolla tube; filaments white. Ovary sessile. Stigma sessile. Capsule 2-valved. Seeds smooth.

15 species: North America, Asia, Europe; four species in Nepal. - Type species: Comastoma tenellum (Rottb.) Toyok.

\section{Key to species of Comastoma}

1a. Stem leaves spathulate or obovate with broad petiole; corolla urn-shaped 
1b. Stem leaves ovate, elliptic or lanceolate, sessile; corolla cylindrical or tubular

2a. Stems weak, straggling; stem leaves ovate, up to $3 \mathrm{~mm}$ long 3. C. stellariifolium

2b. Stems ascending; stem leaves, if present, elliptic or lanceolate, usually more than $3 \mathrm{~mm}$ long 3

3a. Calyx slightly saccate at base, apex of lobes recurved, acuminate 1. C. falcatum

3b. Calyx not saccate at base, lobes erect or spreading, acute 2. C. pedunculatum

1. Comastoma falcatum (Turcz. ex Kar. \& Kir.) Toyok., Bot. Mag. (Tokyo) 74: 198 (1961). - Gentiana falcata Turcz. ex Kar. \& Kir., Bull. Soc. Nat. Mosc. 15: 404 (1842). - Gentiana tenella Turcz. ex Kar. \& Kir. var. falcata C.B.Clarke in Hook.f., Fl. Brit. Ind. 4: 110 (1885). - Gentianella falcata (Turcz. ex Kar. \& Kir.) Harry Sm. in Nilsson, Grana Palyn. 7: 144 (1967). - Type: Baikal, in humidis alpis Nuchusaban, 1834, Turczaninow (lecto LE, designated here, digital image). Fig. 2.

Ascending annual to $20 \mathrm{~cm}$ tall. Stems branching from base. Leaves elliptic, usually clustered at base, stem leaves few, 7-20 × 2-5 mm, acute. Inflorescence

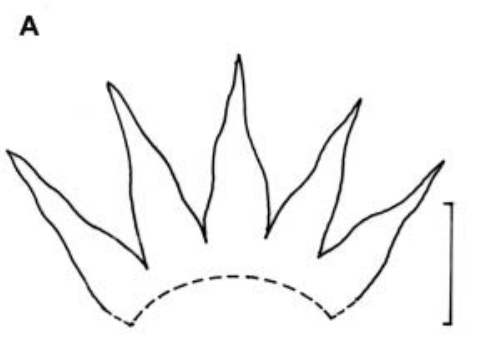

B
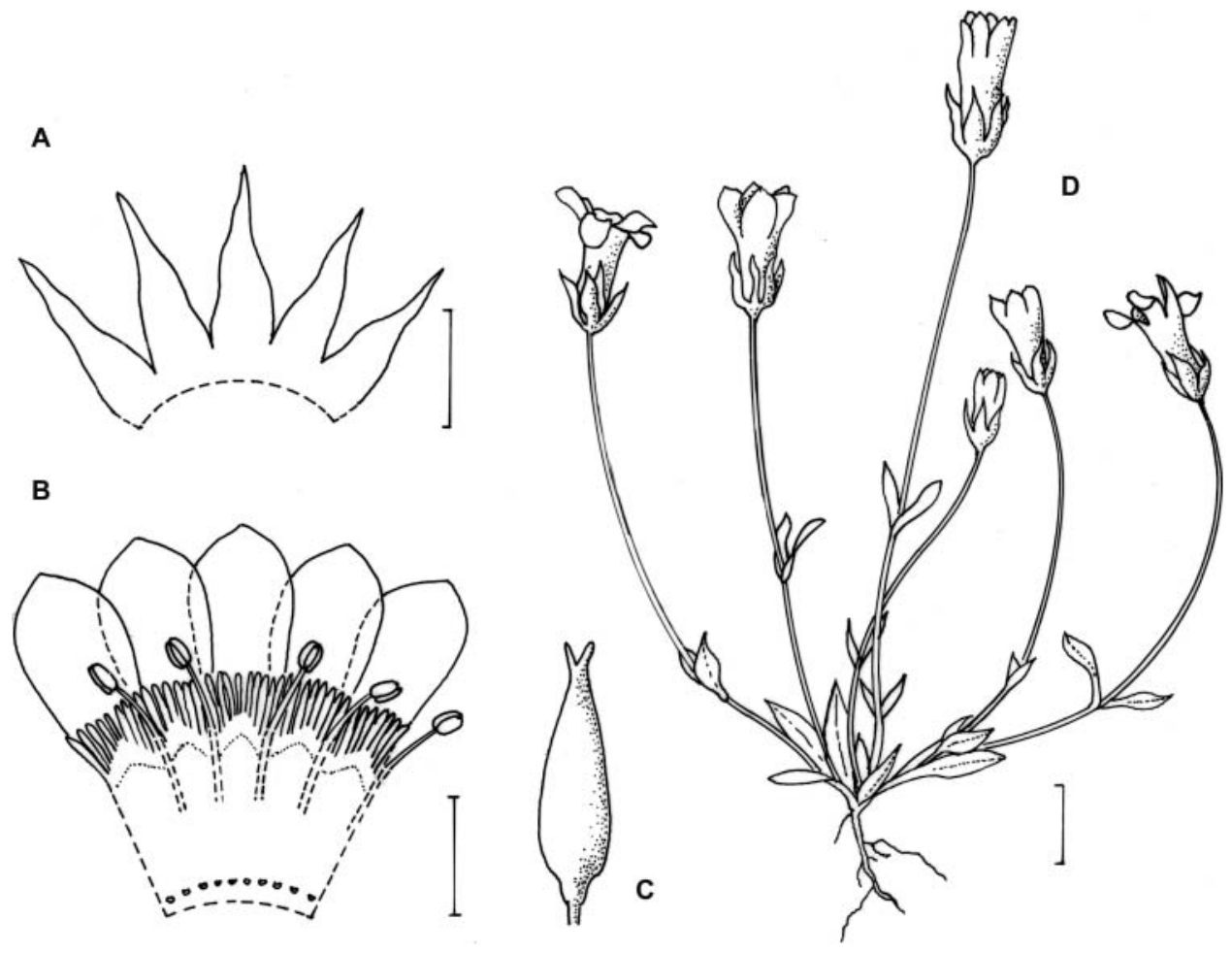

F IG. 2. Comastoma falcatum (Turcz. ex Kar. \& Kir.) Toyok. A, calyx; B, corolla; C, ovary; D, habit. Based on Stainton, Sykes \& Williams 7258. Scale bars: $5 \mathrm{~mm}$ (A-C), $1 \mathrm{~cm}$ (D). 
one-flowered. Pedicel (10-)20-120 mm. Flowers 5-merous. Calyx tube 1-2 mm, slightly saccate at base; lobes unequal, lanceolate, 4-9 × 1-3 mm, apex acuminate, often recurved. Corolla tubular, blue-violet or dark blue; tube 5-10 $\mathrm{mm}$; lobes oblong, 4-8 $\times 2-4 \mathrm{~mm}$, spreading, obtuse or acute. Filaments $3-5 \mathrm{~mm}$; anthers $1-1.5 \mathrm{~mm}$, yellow. Ovary narrowly ellipsoid, $8-10 \times 1-3 \mathrm{~mm}$. Capsule not seen.

Distribution. W, C and E Nepal [Tibetan Plateau (Qinghai, Xinjiang, Xizang); W Himalaya (Pakistan, India, Kashmir); E Asia (China: Gansu, Hebei, Nei Mongol, Shanxi, NW Sichuan); N and C Asia (Mongolia, Siberia, Kyrgyzstan, Tajikistan)].

Ecology. Among stones near streams or on grassy slopes; 4000-5300 m. Flowering July-August.

Specimens examined. W: Dolpa district, Langla Pass between Shimen, Langla and Mur (Mosoc), c.300 m, 6 viii 1973, Grey-Wilson \& Phillips 547 (K). C: Baglung district, Above Dogadi Khola, 15,000 ft, 12 viii 1954, Stainton, Sykes \& Williams 3830 (BM, E). Mustang district, Namdo, north of Mustang, 17,000 ft, 10 viii 1954, Stainton, Sykes \& Williams 2355 (BM); Namdo, north of Mustang, 16,000 ft, 9 viii 1954, Stainton, Sykes \& Williams 2324 (BM); Samargaon, north of Tukucha, 15,000 ft, 16 viii 1954, Stainton, Sykes \& Williams 7258 (BM, E); Jengla, west of Namdo, 5300 m, 25 viii 1973, Grey-Wilson \& Phillips 710 (BM, K); Cha Lungpa, near Sangda-La, 17,200 ft, G. Miehe 137 (BM); Cha Lungpa, 17,200 ft, 14 viii 1977, G. Miehe 472a (BM); Dhaulagiri hidden valley, between Dhampus Pass and French Pass, 5055m, 5 ix-15 x 1980, E. Wald (American Women's Climbing Expedition) 10 (BM). E: Solu Khumbu district, Pheriche, Khumbu, 14,000 ft, 29 viii 1970, Horsfall 34a (BM).

2. Comastoma pedunculatum (D.Don) Holub, Folia Geobot. Phytotax. 3: 218 (1968).

- Eurythalia pedunculata D.Don, London Edinburgh Philos. Mag. \& J. Sci. 8: 76 (1836). - Gentiana pedunculata (D.Don) Royle ex G.Don, Gen. Syst. 4: 182 (1838).

- Gentianella pedunculata (D.Don) Harry Sm. in Nilsson, Grana Palyn. 7: 144 (1967). - Type: Cachemere \& Kunour, Royle 113/12 (lectotype LIV, digital image, plant on far left of sheet designated here). See note below.

Gentiana tenella auct. non Rottb.: C.B.Clarke in Fl. Brit. Ind. 4: 109 (1883).

Nepali name: 'Sermaguru'.

Annual to $22 \mathrm{~cm}$ tall. Stems erect or ascending, branching from base and above. Leaves often all basal; one or two pairs on stems; elliptic to lanceolate, (3-)4-20 $\times$ (1-)2-10 mm, apex rounded or acute. Inflorescence one-flowered or of very loose, few-flowered cymes. Pedicel (10-)15-120 mm. Flowers 5-merous. Calyx tube 1-2 mm; lobes slightly unequal, $(1-) 2-5(-8) \times 1-3 \mathrm{~mm}$, lanceolate or ovate, acute. Corolla bright blue, pale blue, yellow or white; tube cylindrical, often narrow, (4-)8-10 mm; lobes oblong or ovate, $(3-) 4-6(-10) \times 2-3(-4) \mathrm{mm}$, rounded. Filaments $2-3 \mathrm{~mm}$; anthers c. $1 \mathrm{~mm}$. Ovary cylindrical or narrowly ovoid, 4-8 $\times 1-2 \mathrm{~mm}$. Capsule cylindrical, 10-14 $\times 3-5 \mathrm{~mm}$. (Ho \& Pringle (1995) stated that the capsule protrudes from the corolla but this does not seem to be the case with the material from Nepal.) 
Distribution. W, C and E Nepal [Tibetan Plateau (Qinghai, Xizang); W Himalaya (Pakistan, India, Kashmir); E Himalaya (Bhutan, Sikkim); E Asia (China: Gansu, W Sichuan, NW Yunnan)].

Ecology. Open grassy alpine slopes or damp stony ground by rivers; $3050-5100 \mathrm{~m}$. Flowering August-October.

Specimens examined. W: Dolpa district, Rohagaon, Suli Gad, 10,000 ft, 14 ix 1952, Polunin, Sykes \& Williams 3380 (BM, E, K); Rohagaon, Suli Gad, 11,500 ft, 14 ix 1952, Polunin, Sykes \& Williams 3396 (BM, E); North-east of Tiling, c.5100 m, 12 viii 1973, Grey-Wilson \& Phillips 595 (K). Humla district, Saipal, 15,000 ft, 18 viii 1954, J.E.M. Arnold 4 (BM). Jumla district, Chakure Lekh, 14,000 ft, 30 ix 1952, Polunin, Sykes \& Williams 5481 (BM). Mugu district, Tarkia, Mugu Khola, 13,000 ft, 21 viii 1952, Polunin, Sykes \& Williams 5356 (BM, E). C: Baglung district, Above Dogadi Khola, 13,500 ft, 1 ix 1954, Stainton, Sykes \& Williams 4659 (BM). Manang district, Bimtakothi, 13,500 ft, 27 viii 1950, D.G. Lowndes 1471 (BM); Khangsar, 15,000 ft, 9 ix 1950, D.G. Lowndes 1503a (BM, E). Mustang district, Cha Lungpa, 17,200 ft, 14 viii 1977, G. Miehe 472Ab (BM); Cha Lungpa, 17,200 ft, 14 viii 1977, G. Miehe 472Bb (BM) [2 separate sheets]; Tukucha, Kali Gandaki Valley, 12,500 ft, 11 ix 1954, Stainton, Sykes \& Williams 7766c (BM); Tukucha, Kali Gandaki Valley, 12,000 ft, 9 ix 1954, Stainton, Sykes \& Williams 7740 (BM); Tukucha, Kali Gandaki Valley, 12,500 ft, 12 ix 1954, Stainton, Sykes \& Williams 7785 (BM, E); Kimaling, north of Mustang, 14,000 ft, 11 viii 1954, Stainton, Sykes \& Williams 2379 (BM). Rasuwa district, Langtang village area, 11,500 ft, 1 viii 1949, Polunin 1544 (BM, E); Changbu, above Khangyin, $3800 \mathrm{~m}, 21$ ix 1966, D.H. Nicolson 2585 (BM); Langtang Valley, 13,000 ft, 17 ix 1965, J.B. Shrestha \& P.R. Shakya 3886 (BM). E: Sankhuwasabha district, Khumbakarna Himal, Makalu, Upper Barun Valley, 4900 m, 15 ix 1972, T. Wraber 185 (BM); Khumbakarna Himal, Makalu, Upper Barun Valley, 5050 m, 19 ix 1972, T. Wraber 221 (BM); Solu Khumbu, Nangma (Naogma), north of village, W side of Lobuche Khola, 27 $54^{\prime} 54^{\prime \prime} \mathrm{N}, 86^{\circ} 48^{\prime} 53^{\prime \prime} \mathrm{E}$, DNEP3, BX187 (E); Solu Khumbu district, Khumbu, Mt. Everest Base Camp, 16,500 ft, 7 ix 1969, Stainton 6588 (BM); Chule, $27^{\circ} 57^{\prime} 37^{\prime \prime} \mathrm{N}, 86^{\circ} 36^{\prime} 22^{\prime \prime} \mathrm{E}$, DNEP3, AY104 (E). Tapeljung district, Ghunsa, Pung Pema, 5100 m, 9 x 1985, Curzon 217 (K); Kambachen to Lhonak, 4300 m, 10 ix 1989, KEKE 538b (E, K). District uncertain: Dumbudada, 14,600 ft, 5 ix 1932, K.N. Sharma E480 (BM, E).

Notes. The type specimen at LIV was annotated as type material and cited by Omer (1992) but not lectotypified. The evidence for this specimen being original material is circumstantial but probably justified. Although the specimen has not been annotated by David Don, he refers to a Royle collection. Royle himself annotated the specimen as Gentiana pedunculata and the coincidence of the name and the mention of Royle in the protologue would suggest that this is indeed original material.

Clarke (1883) placed Gentiana pedunculata (D.Don) G.Don in synonymy under his description of $G$. tenella. He noted that 'alpine examples half the size of the fully developed plant are frequent'.

3. Comastoma stellariifolium (Franch.) Holub, Folia Geobot. Phytotax. 3: 217 (1968). - Gentiana stellariifolia Franch. in Forbes \& Hems1., J. Linn. Soc. Bot. 26: 135 (1890). - Gentianella stellariifolia (Franch.) Harry Sm. in Nilsson, Grana 
Palyn. 7: 145 (1967). - Type: China: Yunnan, Lopinshan at Langkong, 3200 m, Delavay (holo K).

Gentiana tenella Rottb. var. sikkimensis C.B.Clarke in Hook.f., Fl. Brit. Ind. 4: 110 (1885). - Gentianella tenella (Rottb.) Borner var. sikkimensis (C.B.Clarke) S.Agrawal, Indian Gentianaceae, A Checklist 22 (1988). - Type: Sikkim, iii 1877, Elwes s.n. (lecto K, designated here).

Annual to $10 \mathrm{~cm}$ tall. Stems weak, ascending or straggling. Basal leaves forming rosettes or loose mats, often absent on flowering specimens, lanceolate, 10-15 $\times$ 1-2 mm, acute; stem leaves in widely spaced pairs, ovate, $2-3 \times 1-2 \mathrm{~mm}$, acute. Inflorescence one-flowered. Pedicel 5-40 mm. Flowers 5-merous. Calyx tube $0.5 \mathrm{~mm}$; lobes unequal, lanceolate to elliptic, 3-5 × 1-2 mm, acute. Corolla dark or pale blue; tube 3-6 mm; lobes oblong to ovate, 3-7 $\times 1.5-3 \mathrm{~mm}$, obtuse, spreading. Filaments 1-1.5 mm; anthers c. $0.5 \mathrm{~mm}$. Ovary ellipsoid, 4-7 $\times 1-2 \mathrm{~mm}$. Capsule not exserted from corolla; stipe c. $1 \mathrm{~mm}$.

Distribution. E Nepal [E Himalaya (Bhutan, Sikkim); E Asia (China, NW Yunnan); Assam - Burma (Burma)].

Ecology. Open, sunny, grassy banks; 3530-4300 m. Flowering August-September.

Specimens examined. E: Sankhuwasabha district, Arun Valley, Barun Khola, NW of Num, 13,500 ft, 18 ix 1956, Stainton 1677 (BM); Near Kauma, S of Shipton La, 3530 m, 26 ix 1991, EMAK 283 (E); Sankhuwasabha district, Shipton La, 4030 m, 27 ix 1991, EMAK 341 (E). District uncertain: Tulo Thorme, 4300 m, 23 viii 1972, J.F. Dobremez 1694 (BM).

4. Comastoma urnigerum (E.Aitken \& D.G.Long) E.Aitken, comb. nov. - Gentianella urnigera E.Aitken \& D.G.Long, Edinburgh J. Bot. 53: 82 (1996). - Type: E Nepal: Sankhuwasahba district, S side of Lower Barun Glacier, opposite Mera, $4420 \mathrm{~m}$, 3 x 1991, EMAK 503 (holo E).

Annual to $5 \mathrm{~cm}$ tall. Stems decumbent, diffusely branched from base. Leaves spathulate or obovate, 4-10 $\times 2-4 \mathrm{~mm}$, rounded. Inflorescence one-flowered. Pedicel 3-20 mm. Flowers 4- or 5-merous. Calyx tube 0.5-2 mm; lobes unequal, ovate or lanceolate, 3-5 3 1-3 mm, rounded or acute. Corolla slaty blue or deep blue-violet; tube 3-6 mm; lobes oblong or ovate, $2-4 \times 1.5-2 \mathrm{~mm}$, rounded or subacute. Filaments 1-2 mm; anthers $0.5-1 \mathrm{~mm}$. Ovary ovoid, 3-3.5 × 1-2 mm. Capsule ellipsoid, 5-6 × 2-2.5 mm.

Distribution. E Nepal [E Himalaya (Bhutan, Sikkim); SW China?].

Ecology. Grassy, alpine slopes or stony ground; 4270-4720 m. Flowering and fruiting August-October.

Specimens examined. E: Sankhuwasabha district, $\mathrm{S}$ side of Lower Barun glacier, opposite Mera, 4420 m, 3 x 1991, EMAK 500 (E). 


\section{Doubtful species}

Ohba \& Ikeda (2000) mentioned the following unidentified species of Comastoma.

Comastoma sp. 1: Seto Pokhari - Chhomalang Base Camp, 4500 m (TI 9592268), 4605 m (TI 956297), 4700 m (TI 9584197, 952301); Tangna - Samakang Kharka, $3800 \mathrm{~m}$ (TI 9580385).

Comastoma sp. 2: Khare - Tangna, 4560 m (TI 19584212); around Dudh Kund, $4500 \mathrm{~m}$ (TI 9588235, 9592447), $4610 \mathrm{~m}$ (TI 9584254), $4670 \mathrm{~m}$ (TI 9715229); around Tangna, $4550 \mathrm{~m}$ (TI 9729246); Tangna - Samakang Kharka, $4090 \mathrm{~m}$ (TI 95966419).

This material has not been seen as it is possibly part of a large collection being worked on in Tokyo, but from the description, Comastoma sp. 1 could possibly be $C$. pedunculatum (Royle ex D.Don) Holub. The description of Comastoma sp. 2 is inadequate for identification as no habit, shape or measurements are given. It seems to be unusual in having very short hairs on the upper surface of the leaves. None of the species of Comastoma possess this character and therefore it may belong to another genus.

\section{Gentianopsis Ma, Acta Phytotax. Sin. 1(1): 7 (1951)}

Annual, biennial or perennial, erect herbs. Leaves opposite. Flowers 4-merous, solitary or in loose cymes. Calyx tubular-campanulate, winged or keeled, with triangular, discontinuous intracalycular membrane; 2 outer lobes narrower than, and basally overlapping, the 2 inner lobes. Corolla tubular-campanulate to funnelshaped; margins of lobes entire, toothed or fringed; plicae absent. Nectaries 4, at base of corolla tube. Stamens attached at middle or base of corolla tube. Ovary with distinct stipe, style small or absent, stigmas 2, enlarged. Capsules 2-valved, seeds angular, papillose.

24 species: North America, Asia, Europe; three species in Nepal. - Type species: Gentianopsis barbata (Froel.) Ma.

\section{Key to species of Gentianopsis}

1a. Pedicels 5-30 mm; corolla lobes lacking fringed margins at base 1. G. contorta 1b. Pedicels 25-200 mm; corolla lobes with fringed margins at base

2a. Stem leaves elliptic to lanceolate, $6-15 \mathrm{~mm}$ broad

2. G. paludosa

2b. Stem leaves linear, $1-3 \mathrm{~mm}$ broad 3. G. vvedenskyi

1. Gentianopsis contorta (Royle) Ma, Acta Phytotax. Sin. 1: 14 (1951). - Gentiana contorta Royle, Ill. Bot. Himal. 278, t.68, fig.3 (1835). - Gentianella contorta 
(Royle) Harry Sm. in Hand.-Mazz., Symb. Sin. 7: 979 (1936). - Type: Illustration of Gentiana contorta Royle, Ill. Bot. Himal. 278, t.68, fig.3 (1835) (lecto designated here). Note: Royle states that his specimens from "Mussooree, near Captain Debude's house and the Abbey-hill", from which the drawing was prepared, could not be found. - Epitype: Mussoorie, Lower Park Rd., 6500 ft, x 1915, A. Anderson (epitype E, designated here).

Erect biennial to $32 \mathrm{~cm}$. Stems simple or branching. Lower leaves often withered at flowering; stem leaves elliptic to lanceolate, 15-35 $\times 3-9 \mathrm{~mm}$, acute or rounded. Inflorescence of loose cymes. Pedicel 5-30 mm. Calyx tube 10-17 mm; lobes ovate to lanceolate, 5-8 × 3-5 mm, acuminate. Corolla creamy white; tube 15-18 mm; lobes tightly contorted in bud, pale blue, oblong, 7-8 $\times 2-3 \mathrm{~mm}$, apex rounded, margins not fringed. Filaments c. $10 \mathrm{~mm}$; anthers $0.5 \mathrm{~mm}$. Ovary narrowly ellipsoid, 9-10 $\times$ 3-6 mm; stipe 5-8 mm. Capsule not seen.

Distribution. W and C Nepal [Tibetan Plateau (Qinghai, Xizang); W Himalaya (Pakistan, India); E Himalaya (Bhutan); E Asia (China: Guizhou, Liaoning, Sichuan, Yunnan, Japan)].

Ecology. Dry slopes in coniferous forest; 3000-3100 m. Flowering SeptemberOctober.

Specimens examined. W: Dolpa district, between Rohagaon and Lulo Khola, Suli Gad, 10,000 ft, 15 ix 1952, Polunin, Sykes \& Williams 3401 (BM, E). C: Manang district, Marsyandi Valley, towards Pisang, 3100 m, 24 ix 1969, T. Wraber 394 (BM).

2. Gentianopsis paludosa (Hook.) Ma, Acta Phytotax. Sin. 1(1): 11 (1951). - Gentiana detonsa Rottb. var. paludosa Hook., Hooker's Icon. Pl. 9: t.857 (1852). Gentianella paludosa (Hook.) Harry Sm. in Hand.-Mazz., Symb. Sin. 7: 980 (1936).

- Type: Tibet, marshes at Kisung, Captain Munro 2852 (holo K n.v.). See note below. Fig. 3.

Gentiana detonsa Rottb. var. stracheyi C.B.Clarke in Hook.f., Fl. Brit. Ind. 4: 118 (1883). - Gentianella stracheyi (C.B.Clarke) Harry Sm. in Hand.-Mazz., Symb. Sin. 7: 980 in obs. (1936). - Gentiana stracheyi (C.B.Clarke) Kitam., Fl. Pl. W. Pakistan 117 (1964). - Type: Locality unknown, “Gentiana sp. no. 8”, 26 ix 1848, T. Thomson (lecto $\mathrm{K}$, designated here).

Erect biennial to $55 \mathrm{~cm}$. Stems simple or little-branched, sometimes tinged purple. Basal rosette usually withered at flowering; stem leaves elliptic or lanceolate, 25$45 \times 6-15 \mathrm{~mm}$, rounded or acute. Inflorescence one-flowered or of few-flowered cymes. Pedicel 50-200 mm. Calyx tube 13-18 mm; lobes ovate or lanceolate, 6-12 × 2-7 mm, acute or acuminate. Corolla deep blue, purplish or white, sometimes with thin blue or white stripes; tube $25-40 \mathrm{~mm}$; lobes oblong or spathulate, 8-20 $\times 5-10 \mathrm{~mm}$, margins fringed at base. Filaments $5-15 \mathrm{~mm}$; anthers $1.5-2 \mathrm{~mm}$. Ovary oblong or narrowly ellipsoid, 20-25 × 2-3 mm; stipe 5-15 mm. Capsule 25-35 × $5 \mathrm{~mm}$. 




F IG. 3. Gentianopsis paludosa (Hook.) Ma. A, calyx; B, corolla; C, ovary; D, habit. Based on SHBEN 9470355. Scale bars: $1 \mathrm{~cm}$.

Distribution. W, C and E Nepal [Tibetan Plateau (Qinghai, Xizang); W Himalaya (Pakistan, India); E Himalaya (Bhutan, Sikkim); E Asia (N, NE and SW China)].

Ecology. Dry stony soil, grassy hillsides or by streams; $3420-4880 \mathrm{~m}$. Flowering and fruiting July-September.

Specimens examined. W: Dolpa district, Basia Banyang [Bhanjyang], 4700 m, 6 viii 1973, S. Einarsson, L. Skärby \& B. Wetterhall 2812 (BM); Jarap Khola, 13,000 ft, 17 vii 1966, T.B. Shrestha 5413 (BM); Dolpa, Tarap, $2^{\circ} 2^{\prime} \mathrm{N}, 83^{\circ} 12^{\prime} \mathrm{E}, 13,500 \mathrm{ft}, 17$ vii 1966, Stainton 5548 (BM, E); Barbung Khola, E of Chankkha, c. 4500 m, 31 vii 1973, Grey-Wilson \& Phillips 461 (K); 3 miles north of Shimen, c.4300 m, 6 viii 1973, Grey-Wilson \& Phillips 533 (BM, K). Humla district, Saipal, 15,000 ft, 19 viii 1954, J.E.M. Arnold 70 (BM). Jumla district, Bhurchula Lekhm near Jumla, 12,000 ft, 15 vii 1952, Polunin, Sykes \& Wlliams 4702 (BM). Jumla district?, Maharigaon, 14,000 ft, 22 vii 1953, Polunin, Sykes \& Williams 283 (BM). C: Gorkha district, Thanmanang Kharka, 3700 m, 7 viii 1994, (Suzuki et al.) SHBEN 9485337 (E); Sama Gompa, 3420 m, 8 viii 1994, SHBEN 9470355 (E); Sama Gompa, 3410 m, 8 viii 1994, SHBEN 9485353 (E); Gorkha district, 3420 m, 8 viii 1994, SHBEN 9470355 (2 sheets E). 
Manang district, Marsyandi Khola, near Annapurna II base camp, 12,600 ft, 17 ix 1977, G. Miehe 697 (BM); Sabze Khola, 12,000 ft, 20 vii 1970, D. G. Lowndes 1214 (BM); Marsyandi, 11,500 ft, 13 vii 1950, D. G. Lowndes 1185 (BM, E); Marsyandi, 12,500 ft, 30 vii 1950, D. G. Lowndes 1301 (E); Khangsar, 16,000 ft, 27 vii 1950, D. G. Lowndes 1259 (BM, E); Khangsar, Marsyandi, 12,200 ft, 21 vii 1983, R.J.D. McBeath 1451 (E); Khangsar, Marsyandi, 12,200 ft, 21 vii 1983, R.J.D. McBeath 1452 (E). Mugu district, Khaptang, Mugu Khola, 15,000 ft, 21 viii 1952, Polunin, Sykes \& Williams 5362 (BM, E). Mustang district, 13,000ft, 4 viii 1954, Stainton, Sykes \& Williams 2171 (BM, E); Tanglung, south of Tukucha, Kali Gandaki Valley, 12,500 ft, 15 vii 1954, Stainton, Sykes \& Williams 1785 (BM); Kimaling, north of Mustang, 14,000 ft, 22 viii 1954, Stainton, Sykes \& Williams 2365 (BM, E); Tukucha, Kali Gandaki Valley, 12,500 ft, 11 ix 1954, Stainton, Sykes \& Williams 7757 (BM, E); Muktinath, 15,000 ft, 15 vii 1931, K.N. Sharma E22 (BM); Damodar Kund, north of Muktinath, 14,000 ft, 30 vii 1954, Stainton, Sykes \& Williams 2103 (BM, E); Pura, Muktinath, 12,000 ft, 27 vii 1954, Stainton, Sykes \& Williams 2022 (BM); above Muktinath, 3920 m, 20 viii 1994, SHBEN 9470514 (E); Maharang, south of Mustang, 14,000 ft, 13 viii 1954, Stainton, Sykes \& Williams 7204 (BM, E); Marpa, c.4000 m, 7 ix 1973, Grey-Wilson \& Phillips 804 (K). Rasuwa district, Brangechen Kharka, 12,500 ft, viii 1949, Polunin 1723 (BM); Dagpache, 15,000ft, 13 viii 1949, Polunin 1752 (BM). E: Taplejung district, Tamur Valley,Yangma Khola, NE of Walungchang Gola, 13,000 ft, 23 vii 1956, Stainton 1085 (BM, E). Sankhuwasabha district, Mahagung Kola, 6 miles east of Tingkyu, c.4800 m, 3 viii 1973, Grey-Wilson \& Phillips 484 (BM, K).

Note. Omer (1995: 197) lectotypified the name with the illustration of Gentiana detonsa var. paludosa in Hooker's Icon. Pl. 9: t.857 (1852) as no specimens of Munro 2852 can be found at $\mathrm{K}$.

3. Gentianopsis vvedenskyi (Grossh.) Pissjauk., Fl. Tadzhiksoi S.S.R. 7: 292 (1984). Gentiana vvedenskyi Grossh. in Shishkin \& Bobrov, Fl. URSS 18: 751 (1952). Gentianella vvedenskyi (Grossh.) Harry Sm. in Hara et al., Enum. Fl. Pl. Nepal 3: 94 (1982). - Type: Pamir, Langarsu, in humidis, 17 viii 1857, S. Korshinsky (holo LE, digital image).

Gentiana stricta Klotzsch, Bot. Ergebn. Reise Waldemar 90 (1862), non Willd. ex Schultes (1820). - Type: Hoffmeister (B, probably destroyed). Illustration of $G$. stricta Klotzsch, Bot. Ergebn. Reise Waldemar 90, t.69 (1862) (lecto, designated here). - Epitype: Henus, Ladak, Kashmir, 11,000 ft, 6 viii 1931, W. Koelz 2457 (epitype E, designated here).

Annual or biennial to $45 \mathrm{~cm}$ tall. Stems erect, simple or branched. Basal leaves rosulate, spathulate to lanceolate, $15-40 \times 3-6 \mathrm{~mm}$, usually withered at flowering; stem leaves linear, 6-40 × 1-3 mm, acute, margins revolute. Pedicel 2.5-20 cm. Inflorescence one-flowered or of loose cymes. Calyx tube 7-9 mm; lobes lanceolate, $5-12 \times 2-3 \mathrm{~mm}$, acuminate, with dark prominent mid-vein. Corolla blue; tube 21$28 \mathrm{~mm}$; lobes oblong, 5-9 $\times 2-4 \mathrm{~mm}$, obtuse or rounded, margins fringed at base. Filaments 5-7 mm; anthers oblong, 1-2 mm. Ovary narrowly ellipsoid to cylindrical, c. $15 \times 3 \mathrm{~mm}$; stipe c. $4 \mathrm{~mm}$. Capsule $20-30 \times 3 \mathrm{~mm}$, not protruding from corolla.

Distribution. C Nepal [Tibetan Plateau (Xizang); W Himalaya (Pakistan, India); N Asia (Mongolia); C and SW Asia]. 
Ecology. Open alpine pastures, beside streams; 4150-5000 m. Flowering and fruiting July-September.

Specimens examined. W: Dolpa district, West side of Khung Khola, c.5000 m, 19 viii 1973, Grey-Wilson \& Phillips 679 (K). C: Mustang district, 14,000 ft, 12 viii 1954, Stainton, Sykes \& Williams 2386 (BM); Damodar Kund, north of Muktinath, 14,000 ft, 30 vii 1954, Stainton, Sykes \& Williams 2103A (BM); Chalungpa, 13,600 ft, 29 viii 1977, G. Miehe 554 (BM); Chalungpa, above Jelua Forest, $28^{\circ} 53^{\prime} \mathrm{N}, 83^{\circ} 44^{\prime} \mathrm{E}, 4350 \mathrm{~m}, 6$ ix 2001, G. \& S. Miehe 01-111-04 (E); Thulun Khola, Karak, $29^{\circ} 10^{\prime} \mathrm{N}, 8^{\circ} 56^{\prime} \mathrm{E}, 4080$ m, 22 viii 2001, G. \& S. Miehe 01-060-06 (E); Upper Yakchhu Khola, $28^{\circ} 54^{\prime} \mathrm{N}, 8^{\circ} 55^{\prime} \mathrm{E}, 4500$ m, 7 viii 2001, G. \& S. Miehe 01-020-07 (E).

\section{ACKNOWLEDGEMENTS}

I wish to thank Dr Bernard von Hagen (MJG) for help in defining generic relationships; the Directors of the Herbaria at K and BM for the loan of specimens; Dr Nicholas Hind and Dr Timothy Harris (K-W), Dr John Edmondson (LIV) and Professor Leonid Averyanov (LE) for digital images of type specimens; and Louise Olley for the illustrations.

\section{REFERENCES}

Agrawal, S. (1983). A note on Gentianella glanduligera Airy Shaw (Gentianaceae). Indian Forester 109: 576-577.

Airy Shaw, H. K. (1943). Hooker's Icon. Pl. 35: sub t.3431.

Aitken, E. (1999). Gentianaceae. In: Grierson, A. J . C. \& Long, D. G. (eds) Flora of Bhutan 2(2): 602-656.

Aitken, E. \& Long, D. G. (1994). Notes relating to the Flora of Bhutan: XXVII. Gentianella griersonii, a new species from Bhutan. Edinburgh J. Bot. 51(2): 165-167.

Clarke, C. B. (1883). Gentianaceae. In: Hooker, J. D. (ed.) Flora of British India 4: 93-132.

GARG, S. (1987). Gentianaceae of North West Himalayas (A Revision). International Bioscience Monograph 17. New Delhi: Today and Tomorrow's Printers and Publishers.

von Hagen, K. B. \& Kadereit, J. W. (2001). The phylogeny of Gentianella

(Gentianaceae) and its colonization of the southern hemisphere as revealed by nuclear and chloroplast DNA sequence variation. Organisms Diversity \& Evolution 1: 61-79.

Ho, T. N. \& Pringle, J. S. (1995). Gentianaceae. In: W u, Z. Y. \& R Aven, P. H. (eds) Flora of China 16. Beijing \& St Louis: Science Press \& Missouri Botanical Garden.

Онва, H. \& Ikeda, H. (2000). The Flora of Hinku and Hunku Valleys. Nature and Culture No. 6: 1-272. The University Museum, University of Tokyo.

Omer, S. (1992). Notes on the genus Comastoma Toyok. (Gentianaceae) from Pakistan and Kashmir. Candollea 47: 539-553.

OMer, S. (1995). Gentianaceae. In: A Li, S. I. \& Q Aiser, M. (eds) Flora of Pakistan 197. Karachi, Pakistan: Department of Botany, University of Karachi.

Press, J. R., Shrestha, K. K. \& Sutton, D. A. (2000). Annotated Checklist of the Flowering Plants of Nepal. London: The Natural History Museum. 